\title{
Patient Characteristics and Patterns of Surgical Interventions in Patients of Differentiated Thyroid Cancer with Distant Metastasis
}

\author{
Mohamed Abdul Kathar ${ }^{1}$, Jain Prateek ${ }^{1}$, Kapila Manikantan ${ }^{1}$, Rajeev Sharan ${ }^{1}$, and \\ Pattatheyil Arun ${ }^{1}$ \\ ${ }^{1}$ Tata Medical Center
}

June 8, 2020

\begin{abstract}
Abstract: Unlike most head and neck cancers, the presence of distant metastasis (DM) does not preclude curative intent treatment and surgical interventions are common in metastatic disease. DM has an adverse impact on survival and lends considerable morbidity to the patient. This research attempts to study the demographics, patterns of metastasis, and surgical interventions in this rare subgroup of patients with differentiated thyroid cancer (DTC). Materials and methods: Thirty two patients of DTC with radiologically or histopathologically or cytopathologically confirmed DM who underwent surgery at a tertiary care centre from August 2011 to December 2018 formed the study cohort of this retrospective study. Results: The study population comprised $59 \%$ females and had a median age of 55 (19- 79) years. Thorax was the most common site of metastasis, documented in $56 \%$ of patients, while $53 \%$ patients had bone metastases. Multiple DM were noted in 8 patients. All patients underwent total thyroidectomy or completion thyroidectomy with or without neck dissection. Surgery for metastatic sites was possible in 14 patients (44\%) with debulking and spine fixation being the commonest surgical intervention for metastasis. Thyroidectomy, with or without neck dissection followed by radioactive iodine (RAI) ablation was used as the primary modality to treat DM in patients who did not undergo specific surgical treatment for distant metastases. The median dose of RAI received by patients was $400(25-749) \mathrm{mCi}$, in one to four sessions (median- 2 sessions). Three patients received conventional chemotherapy, while four received Sorafenib. Conclusion: Metastasectomy in differentiated thyroid cancer with distant metastases is feasible in selected patients and surgical interventions are most commonly performed on the spine to prevent neurological complications. RAI ablation is universally administered in this subset of patients and in very high doses, often distributed in multiple sessions. The role of chemotherapy and tyrosine kinase inhibitor is still restricted to palliative settings and cost constraints remain a detriment to more widespread use.
\end{abstract}

Conflicts of interest: The authors claim no competing financial interests

Ethical considerations: This study was registered with our institution's Clinical Audit department. Patient information was anonymised prior to data analysis

\section{Introduction:}

Thyroid malignancy is the fifth most common in women worldwide and it is expected to become the second most common malignancy in women and the ninth most common in men by $2030^{1}$. The incidence of thyroid cancers has been rising over the past few decades. Unlike most head and neck cancers, the presence of distant metastasis (DM) does not preclude curative intent treatment and surgical interventions are common in metastatic disease. DM has an adverse impact on survival and lends considerable morbidity to the patient. Based on the histopathology, thyroid cancers are divided into various subtypes which include differentiated thyroid carcinoma (DTC), medullary and anaplastic thyroid carcinomas. DTCs are derived from thyrocytes, express the sodium transporter and represent $90 \%$ of all thyroid cancers and the terminology encompasses papillary thyroid cancer, follicular carcinoma and poorly differentiated thyroid cancers ${ }^{2}$. 
Genetic determinants of clinical behaviour in DTC are being investigated and find applications in the assessment of the risk of malignancy in indeterminate thyroid nodules ${ }^{3}$. Notable among these markers has been theBRAF ${ }^{\mathrm{V} 600 \mathrm{E}}$ mutation present in $45-59 \%$ of papillary thyroid cancer and associated with a higher incidence of extra thyroidal extension, nodal metastasis, refractoriness to RAI treatment and decreased survival ${ }^{4}$.

Four percent of all patients are diagnosed with or develop distant metastasis. Metastasis develops in 7-15\% of patients following thyroid surgery for DTC ${ }^{5}$. Poorly differentiated thyroid cancers account for $5-10 \%$ of all thyroid cancers and the mean survival after diagnosis is 3.2 years $^{6}$. Bone metastasis have been observed to occur in $2-13 \%$ of patients diagnosed with differentiated thyroid cancers. In thyroid cancer, the overall 10 year survival had been declined to $13-21 \%$ in the presence bone metastasis ${ }^{7}$.

Skeletal survey with whole body bone scan, CT/MRI and whole body MRI in patients with spinal cord involvement is warranted whenever there is a suspicion of metastasis. Biopsy from the metastatic site may be required to confirm the histology ${ }^{8}$.

$\mathrm{I}^{131}$ therapy is the first choice of treatment for papillary and follicular thyroid cancers with distant metastasis, unless they lose the ability to trap iodine ${ }^{9}$. RAI (Radioactive iodine) therapy has shown to be effective in improving the disease free survival in patients diagnosed with locally advanced or metastatic diseases ${ }^{10}$.

According to the guidelines published by the American Thyroid Association (ATA), complete removal of bony metastasis has been seen to exert a beneficial effect on survival and it is recommended particularly in younger patients with spinal metastases with neurological deficits ${ }^{11}$. Hence, completely resectable lesion of bony metastases should be attempted wherever feasible ${ }^{12}$. Lung metastases respond well to radioactive iodine therapy than any other organ metastases but surgical resection of the lung is undertaken only in selected patients, especially with anatomically limited disease ${ }^{13}$.

Tyrosine kinase inhibitors are effective and promising drugs for the treatment of poorly differentiated thyroid cancers $^{14}$. Newer treatment options to treat the locally recurrent and metastatic progressive differentiated thyroid cancers include small molecule oral multi-targeted kinase inhibitors, namely Vandetanib and Sorafenib. Sorafenib works on VEGFR (Vascular endothelial growth factor) 1, 2, and 3 and PDGFR (Platelet derived growth factor).

The role and administration of external beam radiation therapy (EBRT) is not well characterized in differentiated thyroid cancers due to the lack of homogeneity of protocols and conflicting outcomes. In a study done by Lin et al and Benker et $\mathrm{al}^{15}$. They showed that EBRT is not routinely recommended in PTC as there is no significant improvement in overall survival. In fact the higher survival is observed in patients who did not receive EBRT. Despite these drawbacks, in some studies administration of EBRT has improved loco-regional control in invasive disease, unresectable/gross residual tumours and in disease resistant to RAI therapy $^{16}$.

Unlike cancer at other head and neck sites, patients with metastatic DTC frequently undergo surgery during the course of their protracted treatment. There is no available data on the clinical characteristics and patterns of surgical care received by this rare subset of patients leading us to conduct this study.

\section{Materials and methods:}

Data was abstracted from the maintained electronic records of 492 patients with thyroid cancer who were treated surgically at our hospital during the period August 2011 to December 2018. Out of these 492 patients, 381 were diagnosed with differentiated thyroid cancer and 32 patients diagnosed clinically with metastasis from DTC at presentation or who were detected to have metastasis on follow up were included in our analysis. Patients with medullary and anaplastic carcinoma were excluded. The demographics, patterns of metastasis and surgical interventions in this rare subgroup of patients with DTC were studied in this research.

\section{Statistical Analysis:}

Data was analysed using SPSS version 17 (IBM, Chicago IL) 


\section{Results:}

Table 1 shows continuous variables of patient characteristics with metastatic differentiated thyroid cancers

Of the 32 patients with metastatic differentiated thyroid cancer available for final analysis, $59 \%$ were females $(\mathrm{N}=19)$. The median age of the entire study population was 55 years (17-79 years))

Metastatic sites were identified in the thorax (lungs and mediastinum), bones (vertebra, femur, skull, clavicle, manubrium sterni and iliac bones) and brain. Twenty four patients (75\%) had single site metastasis. Metastases were detected at multiple sites in eight patients (25\%). Lung and mediastinal metastases were seen in $56 \%$ and bone metastases were seen in $53 \%$ patients either singly or in combination. One patient had brain metastasis.

Notably, a majority of patients $(\mathrm{N}=20,63 \%)$ had undergone some form of treatment before they were referred to the study centre. Six patients were diagnosed with thyroid cancer after histopathology following surgery to the spine. Four patients had incomplete surgery on the thyroid with our without any intervention for the metastatic disease and one patient had complete surgery for the thyroid but was referred for the care of metastatic disease. The metastatic site was addressed by surgery (metastasectomy) in $44 \%(\mathrm{~N}=14)$. The most common intervention performed was debulking with fixation of the spine which was noted in $15 \%(\mathrm{~N}=5)$. In the remaining 18 patients, the thyroid was ablated with or without a neck dissection and metastatic disease was addressed using radioactive iodine. The majority of cases were diagnosed histologically as papillary thyroid carcinoma $(62.5 \%, \mathrm{~N}=20)$.

Table 2 shows categorical variables of patient characteristics with metastatic differentiated thyroid cancers

\section{Radioactive iodine (RAI) therapy:}

All patients except one patient received adjuvant radioactive iodine therapy in our hospital as one patient received radioactive iodine at the peripheral hospital (RAI). The study population received a maximum of four sessions of treatment with a median of two doses and a cumulative median dose of $400 \mathrm{mCi}$, ranging from $25 \mathrm{mCi}$ to $749 \mathrm{mCi}$.

\section{Systemic therapy:}

Three patients received conventional chemotherapy. One patient received Cisplatin and doxorubicin in our hospital. Two patients received chemotherapy elsewhere and four patients received tyrosine kinase inhibitors. Though Lenvatinib was recommended, all patients received Sorafenib due to cost constraints.

\section{Follow up:}

The median follow up was 26.5 (Range 1-69) months. During this period, there were five deaths in the study cohort. Survival analysis could not be performed due to the paucity of events during follow up and is therefore not being reported.

\section{Discussion:}

Differentiated thyroid cancer carries an excellent prognosis and the development of metastasis is a rare event in everyday head and neck oncology practice. In spite of the evolution of numerous protocols to guide practice, metastases from DTC are treated heterogeneously across treating centres. In our series, metastases were identified in 32 of $381(8.4 \%)$ patients with DTC. The presentation of metastases from DTC is insidious in many cases. In our series $19 \%(\mathrm{~N}=6)$ of patients underwent treatment for conditions in the spine without being aware of primary thyroid pathology. The fact that metastatic workup is not recommended by all established guidelines and that thyroidectomy being a common ENT procedure performed even in low resource non-specialty institutions results in many patients to undergo limited surgeries like hemithyroidectomy and who are subsequently diagnosed with metastases on referral to specialized services after the initial surgery. We had 5 such patients in our series (16\%) The incidence of bone metastasis was $4.5 \%(\mathrm{~N}=17)$ which is in concordance with published literature. Distant metastases are associated with poor prognosis in differentiated thyroid cancers. The most common site of bone metastasis in differentiated thyroid cancers 
are vertebrae, ribs and hips. Tumour cell adhesive molecules bind the tumour cells to marrow stromal cells and bone matrix allowing them to grow and produce angiogenic and bone resorbing factors.

Metastasis of DTC have been treated with curative intent ${ }^{1717}$.In a study done by Isabelle et al, out of 43 patients who had undergone pulmonary metastasectomy predominantly for follicular carcinoma, showed an encouraging 10 year disease free survival of $84 \%$ in patients who underwent complete resection. This significantly declined to $62 \%$ on incomplete resection $(\mathrm{p}=0.013)^{10}$.

In our study, the most common thyroid cancer histopathology was papillary thyroid cancer followed by follicular cancer and poorly differentiated thyroid cancers respectively.

There is uniformity across protocols on the recommendation of radioactive iodine in patients with metastatic DTC in terms of a survival advantage. In a study done on 228 patients, 71 received iodine therapy with a cumulative dose of at least $600 \mathrm{mCi}$, This study reiterated that there seemed to be a better prognosis with greater cumulative doses of RAI but the disease specific mortality was $60 \%$ over a 10 year follow up period when treated with higher cumulative doses ${ }^{11}$, In our study, all except one received radioactive iodine. The median dose of $400 \mathrm{mCi}$ is in accordance with study published by Pitoia et al which showed that the patient treated with a mean effective cumulative RAI dose of $457.3 \pm 29.7 \mathrm{mCi} \mathrm{I}{ }^{131}$ (300-600 $\mathrm{mCi}$ ) had a good response to treatment ${ }^{18}$.

It is imperative that young patients with metastatic DTC be warned of the adverse effects of high dose radioiodine, most importantly xerostomia, pulmonary fibrosis and infertility. The option of cryopreservation of semen or oocytes may be offered to potential recipients of high dose radioactive iodine ${ }^{19}$.

The role of conventional EBRT has been well established in the literature to allay pain, to improve neurological deficits and or to prevent pathological fractures. Four patients in our series received EBRT, all for the alleviation of pain. Studies show that radiation doses vary from low risk microscopic disease (54 Gy), High risk (59.5 Gy) to gross disease (63-70 Gy) to achieve local control rates ${ }^{20}$. However, EBRT has been found to show a higher rate of local progression and pain relapse with long term follow up ${ }^{21}$. In our research, only four patients received EBRT to the metastatic site with a maximum of 54 Gy and a minimum of 30 Gy.

\section{Conclusion:}

Surgical interventions are common in patients with metastatic DTC. Procedures to the neck commonly include completion of thyroidectomy and clearance of nodes from the lateral neck, Procedures to the metastatic sites include debulking and fixation of the spine and metastasectomy. A high index of suspicion is needed while evaluating spinal lesions and the possibility of metastasis from the thyroid needs to be entertained. All patients receive radioactive iodine in conjunction with surgery in high doses with its attendant complications. Most patients receive a combination of therapies in varied sequences and the goal of treatment should be to mitigate complications and improve quality of life since most patients live long in spite of their advanced disease. The role of radiotherapy needs to be defined in large multicentre trials and more experience with targeted therapy is needed to formulate robust guidelines.

\section{Limitations:}

This is a single centre experience and in spite of the collation of data over seven years, we could collect information from 32 patients. Large collaborative data sets would be necessary to throw light on this issue. The implications of various patterns of metastases on survival would have provided interesting insights but we were constrained by the fact that even with a long period of follow up, only five events were documented.

\section{References}

1. Ullmann TM, Gray KD, Moore MD, Zarnegar R, Fahey Iii TJ. Current controversies and future directions in the diagnosis and management of differentiated thyroid cancers. Gland Surgery. 2018;7(5):473-486.

2. Schmidbauer B, Menhart K, Hellwig D, Grosse J. Differentiated Thyroid Cancer-Treatment: State of the Art. IJMS. 2017;18(6):1292. 
3. McLeod DSA. Current Concepts and Future Directions in Differentiated Thyroid Cancer. Clin Biochem Rev. 2010;31(1):9-19.

4. Li F, Chen G, Sheng C, et al. BRAFV600E mutation in papillary thyroid microcarcinoma: a metaanalysis.Endocrine-Related Cancer. 2015;22(2):159-168.

5. Siegel R, Ma J, Zou Z, Jemal A. Cancer statistics, 2014. CA: A Cancer Journal for Clinicians.2014;64(1):929.

6. Landa I, Ibrahimpasic T, Boucai L, et al. Genomic and transcriptomic hallmarks of poorly differentiated and anaplastic thyroid cancers. Journal of Clinical Investigation.2016;126(3):1052-1066.

7. Elshafie OT, Hussein S, Al-Hamdani A, Bererhi H, Woodhouse NJY. Multiple Bone Metastases in a Patient with Differentiated Thyroid Cancer (DTC). Sultan Qaboos Univ Med J.2010;10(1):101-105.

8. Wexler JA. Approach to the Thyroid Cancer Patient with Bone Metastases. The Journal of Clinical Endocrinology $\mathcal{E}$ Metabolism. 2011;96(8):2296-2307.

9. Lin J-D, Kuo S-F, Huang B-Y, Lin S-F, Chen S-T. The efficacy of radioactive iodine for the treatment of well-differentiated thyroid cancer with distant metastasis. Nucl Med Commun. 2018;39(12):1091-1096.

10. Singh Ospina N, Castro MR. Treatment of Recurrent/Metastatic Thyroid Cancer with Radioactive Iodine. In: Roman SA, Sosa JA, Solórzano CC, eds. Management of Thyroid Nodules and Differentiated Thyroid Cancer: A Practical Guide.Cham: Springer International Publishing; 2017:315-329.

11. Kato S, Murakami H, Demura S, et al. The impact of complete surgical resection of spinal metastases on the survival of patients with thyroid cancer. Cancer Med.2016;5(9):2343-2349.

12. Kushchayeva YS, Kushchayev SV, Wexler JA, et al. Current Treatment Modalities for Spinal Metastases Secondary to Thyroid Carcinoma. Thyroid. 2014;24(10):1443-1455.

13. Durante C, Haddy N, Baudin E, et al. Long-Term Outcome of 444 Patients with Distant Metastases from Papillary and Follicular Thyroid Carcinoma: Benefits and Limits of Radioiodine Therapy. The Journal of Clinical Endocrinology \& Metabolism. 2006;91(8):2892-2899.

14. Sherman SI. Early clinical studies of novel therapies for thyroid cancers. Endocrinol Metab Clin North Am. 2008;37(2):511-524, xi.

15. Lee N, Tuttle M. The role of external beam radiotherapy in the treatment of papillary thyroid cancer.Endocrine-Related Cancer. 2006:971-977.

16. Kiess AP, Agrawal N, Brierley JD, et al. External-beam radiotherapy for differentiated thyroid cancer locoregional control: A statement of the American Head and Neck Society.Head Neck. 2016;38(4):493-498.

17. Porterfield JR, Cassivi SD, Wigle DA, et al. Thoracic metastasectomy for thyroid malignancies.European Journal of Cardio-Thoracic Surgery. 2009;36(1):155-158.

18. Pitoia F, Bueno F, Cross G. Long-Term Survival and Low Effective Cumulative Radioiodine Doses to Achieve Remission in Patients With 131Iodine-Avid Lung Metastasis From Differentiated Thyroid Cancer. Clinical Nuclear Medicine.2014;39(9):784-790.

19. Ceccarelli C, Canale D, Vitti P. Radioactive iodine (131I) effects on male fertility. Current Opinion in Urology. 2008;18(6):598-601.

20. Turaka A, Li T, Yu JQ, et al. Intensity Modulated Radiation Therapy (IMRT) for Thyroid Cancer.8.

21. Yarnold JR. 8 Gy single fraction radiotherapy for the treatment of metastatic skeletal pain: randomised comparison with a multifraction schedule over 12 months of patient follow-upOn behalf of the Bone Pain Trial Working Party.Radiotherapy and Oncology. 1999;52(2):111-121.

Figure Legends: 
Table 1:

\begin{tabular}{lll}
\hline Characteristic & Median & Range \\
\hline Age & 55 & $17-79$ \\
No. of Metastatic sites & 1 & $1-5$ \\
RAI Dose & 400 & $25-749$ \\
RAI Sessions & 2 & $0-4$ \\
\hline
\end{tabular}

$(\mathrm{RAI}=$ radioactive Iodine, $\mathrm{RAI}$ Dose in $\mathrm{mCi})$

Table 2:

\begin{tabular}{ll}
\hline Characteristic & Percent (N) \\
\hline Gender Female Male & $59(19) 41(13)$ \\
Single Metastatic Site & $75(24)$ \\
Thoracic Metastasis & $56(18)$ \\
Bone Metastasis & $53(17)$ \\
Multiple metastasis & $25(8)$ \\
Metastasectomy & $44(14)$ \\
Initial treatment & $63(20)$ \\
Histopathology & \\
Papillary carcinoma & $63(20)$ \\
Follicular carcinoma & $28(9)$ \\
Poorly Differentiated & $9(3)$ \\
\hline
\end{tabular}

\section{Hosted file}

Tables 07.06.20.docx available at https://authorea.com/users/331063/articles/457796-patientcharacteristics-and-patterns-of-surgical-interventions-in-patients-of-differentiatedthyroid-cancer-with-distant-metastasis 